\title{
EXTENSIONS IN BOUNDED TOPOLOGY
}

\author{
DIETER LESEBERG
}

\begin{abstract}
In a series of papers, Leseberg has studied and examined several fundamental aspects of generalized nearness in the realm of bounded topology. This paper deals with an alternate, detailed description of so-called bounded nearness and bounded topology by certain subdensity spaces and related maps. This type of spaces contains, in particular, $b$-near spaces as well as $b$-topological spaces in a nice fashion. Moreover, we found a one-to-one correspondence between suitable subdensity spaces and the related strict topological extensions. We point out that this newly established connection generalize the extensions which were studied by Bentley, Hušek, Ivanova, Ivanov, Lodato and Smirnov.
\end{abstract}

\section{INTRODUCTION}

In the paper, we study b-nearness spaces as well as b-topological spaces in the realm of bounded topology. These spaces can be nicely described by the socalled subdensity spaces and corresponding maps. Thus, they may be considered as generalizations of some classical concepts like quasi-nearness spaces or quasitopological spaces [11]. In this regard, we will find a one-to-one correspondence between suitable subdensity spaces and the related strict topological extensions. Thus, the newly established connection will generalize the extensions which were studied by Bentley [3], Hušek [9], Ivanova and Ivanov [10], Lodato [19] and Smirnov $[22]$.

\section{BASIC CONCEPTS}

As usual, $\underline{P} X$ denotes the power set of a set $X$, and we call a subset $\mathcal{B}^{X} \subseteq \underline{P} X$ a boundedness (on $X$ ) if it possesses the following properties:

$\left(\mathrm{b}_{0}\right) \emptyset \in \mathcal{B}^{X}$

(b) $B_{2} \subseteq B_{1} \in \mathcal{B}^{X}$ imply $B_{2} \in \mathcal{B}^{X}$;

$\left(\mathrm{b}_{2}\right) x \in X$ implies $\{x\} \in \mathcal{B}^{X}$.

The elements of $\mathcal{B}^{X}$ are called bounded sets. Given boundednesses $\mathcal{B}^{X}$ and $\mathcal{B}^{Y}$, a function $f: X \longrightarrow Y$ is called bounded if it satisfies the condition

(b) $B \in \mathcal{B}^{X}$ implies $f[B] \in \mathcal{B}^{Y}$.

$M S C$ (2010): primary 54A05, 54B30, 54C20, 54D35, 54D80, 54E17.

Keywords: bounded topology, $b$-topological space, $b$-nearness space, bornotopological extension.

This paper is dedicated to my dear colleague Harry Poppe on the occasion of his 81st birthday and in addition to my very good friend René Bartsch. 
We denote by BOUND the corresponding category and mention here its convenient properties that make it a topological universe. Further, we call a bounded function bi-bounded if it is, moreover, rebounded, i.e., satisfies

(rb) $B \in \mathcal{B}^{Y}$ implies $f^{-1}[B] \in \mathcal{B}^{X}$.

Then, we introduce the following denotation: For subsets $\rho, \rho_{1}, \rho_{2} \subseteq \underline{P} X$, we put:

$$
\begin{aligned}
& \rho_{2}<<\rho_{1} \text { if and only if } \forall F_{2} \in \rho_{2} \exists F_{1} \in \rho_{1} F_{1} \subseteq F_{2} ; \\
& \rho_{1} \vee \rho_{2}:=\left\{F_{1} \cup F_{2}: F \in \rho_{1}, F_{2} \in \rho_{2}\right\} ; \\
& \bigcap \rho:=\bigcap\{F \subseteq X: F \in \rho\} .
\end{aligned}
$$

Definition 2.1. We call a triple $\left(X, \mathcal{B}^{X}, N\right)$ consisting of a set $X$, boundedness $\mathcal{B}^{X}$ and a function $\left.N: \mathcal{B}^{X} \longrightarrow \underline{P}(\underline{P}(\underline{P} X))\right)$ a subdensity space (shortly sd-space) if the following axioms are satisfied:

$\left(\operatorname{sd}_{1}\right) B \in \mathcal{B}^{X}$ implies $c l_{N}(B) \in \mathcal{B}^{X}$, where in general $c l_{N}(B):=\{x \in X:\{B\} \in$ $N(\{x\})\}$

$\left(\operatorname{sd}_{2}\right)$ the conditions $\rho \subseteq \underline{P} X, \rho \cap \mathcal{B}^{X} \in N(B)$ and $B \in \mathcal{B}^{X} \backslash\{\emptyset\}$ imply $\rho \in N(B)$;

$\left(\operatorname{sd}_{3}\right)$ the conditions $\rho_{2}<<\rho_{1} \in N(B), B \in \mathcal{B}^{X}$ and $\rho_{2} \subseteq \underline{P} X$ imply $\rho_{2} \in N(B)$;

$\left(\operatorname{sd}_{4}\right) B \in \mathcal{B}^{X}$ implies $\mathcal{B}^{X} \notin N(B) \neq \emptyset$;

$\left(\operatorname{sd}_{5}\right) \rho \in N(\emptyset)$ implies $\rho=\emptyset$;

$\left(\operatorname{sd}_{6}\right) x \in X$ implies $\{\{x\}\} \in N(\{x\})$;

$\left(\operatorname{sd}_{7}\right) \emptyset \neq B_{2} \subseteq B_{1} \in \mathcal{B}^{X}$ imply $N\left(B_{2}\right) \subseteq N\left(B_{1}\right)$;

$\left(\operatorname{sd}_{8}\right)\left\{c l_{N}(F): F \in \rho\right\} \in N(B), \rho \subseteq \underline{P} X, B \in \mathcal{B}^{X}$ imply $\rho \in N(B)$.

Given subdensity spaces $\left(X, \mathcal{B}^{X}, N\right)$ and $\left(Y, \mathcal{B}^{Y}, M\right)$, a bi-bounded function $f$ : $X \longrightarrow Y$ is called a density map (shortly $d$-map) if it satisfies

(d) $B \in \mathcal{B}^{X}$ and $\rho \in N(B)$ imply $f \rho:=\{f[F]: F \in \rho\} \in M(f[B])$.

We denote by SD the category of $s d$-spaces and $d$-maps.

Remark 2.2. Subdensity spaces are special cases of a density space, which is called hypernear space in [17]. Then, nearness and topology may be considered as a certain kind of so-called saturated hypernear spaces. We will extend the concepts of topology and nearness by deleting the requirement of saturation to obtain socalled b-quasinearness and b-quasitopologies, respectively. It will be shown that both of these fundamental notions can be described by suitable subdensity spaces in a nice fashion.

Remark 2.3. As already observed, hypertopologies or, equivalently, quasitopologies appear in connection with certain interior operators studied by Kent and Min [11]. Hereby, a function $h: \underline{P} X \longrightarrow \underline{P} X$ is called a quasitopological operator on $X$, and the pair $(X, h)$ is called a quasitopological space (shortly a qtopological space) if $h$ satisfies the following conditions:

$\left(\mathrm{h}_{1}\right) h(\emptyset)=\emptyset$

(h) $A \in \underline{P} X$ implies $A \subseteq h(A)$;

$\left(\mathrm{h}_{3}\right) A_{1} \subseteq A_{2} \in \underline{P} X$ imply $h\left(A_{1}\right) \subseteq h\left(A_{2}\right)$;

$\left(\mathrm{h}_{4}\right) \quad A \in \underline{P} X$ implies $h(h(A)) \subseteq h(A)$.

Given quasitopological spaces $\left(X, h_{1}\right),\left(Y, h_{2}\right)$, a function $f: X \longrightarrow Y$ is called continuous if it satisfies the following property:

(c) $A \subseteq \underline{P} X$ implies $f\left[h_{1}(A)\right] \subseteq h_{2}(f[A])$. 
We denote by QTOP the category of qtopological spaces and continuous functions. Analogously, it is possible to consider quasinearness spaces defined by the below listed properties.

For a set $X, \xi \in \underline{P}(\underline{P}(\underline{P} X)))$ is called a quasinearness (on $X)$, and the pair $(X, \xi)$ is called a quasinearness space (shortly qnear space) if the following axioms hold:

$\left(\mathrm{n}_{1}\right) \mathcal{A}_{2}<<A_{1} \in \xi$ and $\mathcal{A}_{2} \subseteq \underline{P} X$ imply $\mathcal{A}_{2} \in \xi$;

$\left(\mathrm{n}_{2}\right)\{\emptyset\} \notin \xi$ and $\emptyset \in \xi$;

$\left(\mathrm{n}_{3}\right) \cap \mathcal{A} \neq \emptyset$ implies $\mathcal{A} \in \xi$;

$\left(\mathrm{n}_{4}\right)\left\{c l_{\xi}(A): A \in \mathcal{A}\right\} \in \xi, \mathcal{A} \subseteq \underline{P} X$ imply $\mathcal{A} \in \xi$, where $c l_{\xi}(A):=\{x \in X:$ $\{\{x\}, A\} \in \xi\}$.

The nearness preserving maps are defined in an obvious way. We denote by QNEAR the category of qnear spaces and nearness preserving maps. Let us mention the fact that each symmetric qtopological space $(X, h)$ induces a qnear space $\left(X, \xi_{h}\right)$ by setting:

$$
\xi_{h}:=\{\mathcal{A} \in \underline{P} X: \bigcap\{h(A): A \in \underline{P} X\} \neq \emptyset\} .
$$

In this connection, we recall that $h$ is symmetric if it satisfies the corresponding condition

(s) for all $x, z \in X, x \in h(\{z\})$ implies $z \in h(\{x\})$.

So it is possible to describe completely symmetric qtopological spaces by their corresponding qnear spaces (see also [7]). Now, we will extend the above concepts of quasitopology and quasinearness to more general settings by introducing bounded nearness and bounded topology, respectively.

Definition 2.4. Let $\mathcal{B}^{X}$ be a boundedness on $X$. A function $h: \mathcal{B}^{X} \longrightarrow \underline{P} X$ is called a b-quasitopological operator (shortly b-qtopology) (on $X$ ) if the following axioms are satisfied:

(b-t t $\left._{1}\right) B \in \mathcal{B}^{X}$ implies $h(B) \in \mathcal{B}^{X}$;

$\left(\mathrm{b}-\mathrm{t}_{2}\right) h(\emptyset)=\emptyset$;

(b-t t $\left._{3}\right) B \in \mathcal{B}^{X}$ implies $B \subseteq h(B)$;

(b-t t $) B_{1} \subseteq B_{2} \in \mathcal{B}^{X}$ imply $h\left(B_{1}\right) \subseteq h\left(B_{2}\right)$;

(b-t b $_{5} B \in \mathcal{B}^{X}$ implies $h(h(B)) \subseteq h(B)$.

Then, the triple $\left(X, \mathcal{B}^{X}, h\right)$ is called a b-qtopological space.

For b-qtopological spaces $\left.\left(X, \mathcal{B}^{X}, h_{1}\right)\right),\left(Y, \mathcal{B}^{Y}, h_{2}\right)$, a bi-bounded function $f$ : $X \longrightarrow Y$ is called b-continuous if it satisfies the condition

(b-c) $B \in \mathcal{B}^{X}$ implies $f\left[h_{1}(B)\right] \subseteq h_{2}(f[B])$.

We denote by b-QTOP the category of b-qtopological spaces and b-continuous functions.

Remark 2.5. For each qtopological space $(X, h),(X, \underline{P} X, h)$ is a b-qtopological space. Moreover, if $\mathcal{B}^{X}$ is a boundedness, then the set $\mathcal{B}_{h}^{X}:=\{D \subseteq X: \exists B \in$ $\left.\mathcal{B}^{X} D \subseteq h(B)\right\}$ is a boundedness too. Thus, $\left(X, \mathcal{B}_{h}^{X}, h^{b}\right)$ defines a b-qtopological space where $h^{b}:=\left.h\right|_{\mathcal{B}_{h}^{X}}$ is the restriction of $h$ to $\mathcal{B}_{h}^{X}$.

Definition 2.6. Let $\mathcal{B}^{X}$ be a boundedness on a set $X$. A subset $\xi \subseteq \underline{P \mathcal{B}^{X}}$ is called a $b$-quasinearness and the triple $\left(X, \mathcal{B}^{X}, \xi\right)$ is called a $b$-quasinearness space (shortly $b$-qnear space) if following axioms are valid: 
(b-n n $_{1} B \in \mathcal{B}^{X}$ implies $c l_{\xi}(B) \in \mathcal{B}^{X}$, where $c l_{\xi}(B):=\{x \in X:\{\{x\}, B\} \in \xi\} ;$

$\left(\mathrm{b}-\mathrm{n}_{2}\right) \mathcal{A}_{2}<<\mathcal{A}_{1} \in \xi$ and $\mathcal{A}_{2} \in \underline{P} \mathcal{B}^{X}$ imply $\mathcal{A}_{2} \in \xi$

$\left(\mathrm{b}-\mathrm{n}_{3}\right)\{\emptyset\} \notin \xi$ and $\emptyset \in \xi$;

(b-n $\left.{ }_{4}\right) \quad x \in X$ implies $\{\{x\}\} \in \xi$;

(b-n $\left.\mathrm{n}_{5}\right)\left\{\operatorname{cl}_{\xi}(A): A \in \mathcal{A}\right\} \in \xi$ and $\mathcal{A} \in \underline{P \mathcal{B}^{X}}$ imply $\mathcal{A} \in \xi$.

For b-qnear spaces $\left(X, \mathcal{B}^{X}, \xi\right),\left(Y, \mathcal{B}^{Y}, \eta\right)$, a bi-bounded function $f: X \longrightarrow Y$ is called a $b$-near map (shortly a $b$-nmap) if it satisfies the condition

(b-n) $A \in \xi$ implies $f \mathcal{A} \in \xi$.

We denote by b-QNEAR the category of b-qnear spaces and b-nmaps.

Remark 2.7. For each qnear space $(X, \xi),(X, \underline{P} X, \xi)$ is a b-qnear space. Moreover, if $\mathcal{B}^{X}$ is a boundedness, then $\left(X, \mathcal{B}_{c l_{\xi}}^{X}, \xi^{b}\right)$ is a b-qnear space where $\xi^{b}:=\left\{\rho \in \underline{P B}_{c l_{\xi}}^{X}: \rho \in \xi\right\}$. Let us mention the fact that each symmetric bqtopological space $\left(X, \mathcal{B}^{X}, h\right)$ induces a b-qnear space $\left(X, \mathcal{B}^{X}, \xi_{h}\right)$ by setting:

$$
\left.\xi_{h}:=\left\{\mathcal{A} \in \underline{P B}^{X}: \bigcap\{h(A): A \in \mathcal{A}\} \neq \emptyset\right\}\right\}
$$

(compare with Remark 2.3). This connection generalizes the classical one, see [7].

\section{IMPORTANT ISOMORPHISMS}

First, we will find a full embedding of the category b-QTOP into SD:

Proposition 3.1. For a b-qtopological space $\left(X, \mathcal{B}^{X}, h\right)$, we set $N_{h}(\emptyset):=\{\emptyset\}$ and $N_{h}(B):=\left\{\rho \subseteq \underline{P} X: B \cap\left(\cap\left\{h(F): F \in \rho \cap \mathcal{B}^{X}\right\}\right) \neq \emptyset\right\}$ if $B \neq \emptyset$. Then, $\left(X, \mathcal{B}^{X}, N_{h}\right)$ is a subdensity space so that $h=c l_{N_{h}}^{b}$, where $c l_{N_{h}}^{b}$ denotes the restriction of $c l_{N_{h}}$ to $\mathcal{B}^{X}$.

Proof. $\quad\left(\mathrm{sd}_{1}\right)$ : For $B \in \mathcal{B}^{X}$, we have $h(B) \in \mathcal{B}^{X}$ according to (b-t $\left.\mathrm{t}_{1}\right)$. It remains to verify that $c l_{N_{h}}(B) \subseteq h(B)$. But $x \in c l_{N_{h}}(B)$ implies $\{B\} \in N_{h}(\{x\})$, hence, $x \in h(B)$ because $\{B\} \cap \mathcal{B}^{X}=\{B\}$.

$\left(\operatorname{sd}_{2}\right)$ : For $\rho \subseteq \underline{P} X$ and $B \in \mathcal{B}^{X} \backslash\{\emptyset\}$, let $\rho \cap \mathcal{B}^{X} \in N_{h}(B)$. Hence, there exists $x \in B$ with $x \in \bigcap\left\{h(F): F \in\left(\rho \cap \mathcal{B}^{X}\right) \cap \mathcal{B}^{X}\right\}$. Consequently, $x \in$ $\bigcap\left\{h(F): F \in \rho \cap \mathcal{B}^{X}\right\}$ follows, which yields $\rho \in N_{h}(B)$.

$\left(\operatorname{sd}_{3}\right)$ : Let $B \in \mathcal{B}^{X} \backslash\{\emptyset\}$ and $\rho_{2}<<\rho_{1} \in N_{h}(B), \rho_{2} \subseteq \underline{P} X$. By the hypothesis, we can find an element $x \in B$ with $x \in \bigcap\left\{h\left(F_{1}\right): F_{1} \in \rho_{1} \cap \mathcal{B}^{X}\right\}$. For $F_{2} \in \rho_{2} \cap \mathcal{B}^{X}$, there exists $F_{1} \in \rho_{1}$ such that $F_{1} \subseteq F_{2}$, hence, $F_{1} \in \rho_{1} \cap \mathcal{B}^{X}$ is valid, and $x \in h\left(F_{1}\right) \subseteq h\left(F_{2}\right)$ results implying $\rho_{2} \in N_{h}(B)$.

$\left(\operatorname{sd}_{4}\right)-\left(\operatorname{sd}_{7}\right)$ : are clear.

$\left(\operatorname{sd}_{8}\right)$ : Let $B \in \mathcal{B}^{X} \backslash\{\emptyset\}$ and $\left\{c l_{N_{h}}(F): F \in \rho\right\} \in N_{h}(B), \rho \subseteq \underline{P} X$. We have to show that $\rho \in N_{h}(B)$. By the hypothesis, we can choose $x \in B$ with $x \in \bigcap\left\{h(A): A \in\left\{c l_{N_{h}}(F): F \in \rho\right\} \cap \mathcal{B}^{X}\right\}$. Now, let $F \in \rho \cap \mathcal{B}^{X}$. Then, we get $x \in h\left(c l_{N_{h}}(F)\right)$. It remains to verify that $c l_{N_{h}}(F) \subseteq h(F)$ because, then, $x \in h(F)$ is valid. $z \in c l_{N_{h}}(F)$ implies $\{F\} \in N_{h}(\{z\})$ and, consequently, $z \in h(F)$ because $\{F\} \cap \mathcal{B}^{X}=\{F\}$. To prove the equation $h=c l_{N_{h}}^{b}$, let $x \in h(B)$ for $B \in \mathcal{B}^{X}$. Then, $\{B\} \in N_{h}(\{x\})$ and thus $x \in c l_{N_{h}}(B)$, which means $x \in c l_{N_{h}}^{b}(B)$. Conversely, $x \in c l_{N_{h}}^{b}(B)$ implies $x \in \operatorname{cl}_{N_{h}}(B)$, hence, $\{B\} \in N_{h}(\{x\})$. Consequently, $x \in h(B)$. 
Remark 3.2. By the definition of $N_{h}$, the subdensity space $\left(X, \mathcal{B}^{X}, N_{h}\right)$ has an additional property, which we will now take into account.

Definition 3.3. Let us call a subdensity space $\left(X, \mathcal{B}^{X}, N\right)$ qtopological if $N$ possesses the following property:

(qt) $B \in \mathcal{B}^{X} \backslash\{\emptyset\}$ and $\rho \in N(B)$ imply the existence of $x \in \mathcal{B}$ with $x \in$ $\bigcap\left\{c l_{N}(F): F \in \rho \cap \mathcal{B}^{X}\right\}$.

We denote by QT-SD the full subcategory of SD whose objects are the qtopological spaces.

Remark 3.4. Let us note that, given a qtopological space $\left(X, \mathcal{B}^{X}, N\right)$, the triple $\left(X, \mathcal{B}^{X}, c l_{N}^{b}\right)$ defines a b-qtopological space where $c l_{N}^{b}$ denotes the restriction of $c l_{N}$ to $\mathcal{B}^{X}$.

Proposition 3.5. For a qtopological subdensity space $\left(Y, \mathcal{B}^{Y}, M\right)$, the triple $\left(Y, \mathcal{B}^{Y}, c l_{M}^{b}\right)$ is a b-qtopological space such that $M=N_{c l_{M}^{b}}$.

Proof. $\left(Y, \mathcal{B}^{Y}, c l_{M}^{b}\right)$ defines a b-qtopological space according to Remark 3.4. To prove the above mentioned equality, let $\rho \in N_{c l_{M}^{b}}(B)$ and $B \neq \emptyset$. We can choose $x \in B$ with $x \in \bigcap\left\{c l_{M}^{b}(F): F \in \rho \cap \mathcal{B}^{X}\right\}$. By $\left(\operatorname{sd}_{6}\right),\{\{x\}\} \in M(\{x\})$ and $\left\{c l_{M}(F): F \in \rho \cap \mathcal{B}^{X}\right\}<<\{\{x\}\}$. According to $\left(\operatorname{sd}_{3}\right),\left\{c l_{M}(F): F \in \rho \cap \mathcal{B}^{X}\right\} \in$ $M(\{x\})$ and $\rho \cap \mathcal{B}^{X} \in M(\{x\})$ is valid with respect to $\left(\operatorname{sd}_{8}\right)$. But $M(\{x\}) \subseteq M(B)$ holds by $\left(\mathrm{sd}_{7}\right)$, and so, $\rho \cap \mathcal{B}^{X} \in M(B)$. By applying $\left(\operatorname{sd}_{2}\right)$, we get $\rho \in M(B)$. Conversely, let $\rho \in M(B)$. Since $M$ satisfies (qt), we can choose $x \in B$ with $x \in \bigcap\left\{c l_{M}(F): F \in \rho \cap \mathcal{B}^{X}\right\}$. Consequently, $x \in \bigcap\left\{c l_{M}^{b}(F): F \in \rho \cap \mathcal{B}^{X}\right\}$ so that $\rho \in N_{c l_{M}^{b}}(B)$.

Theorem 3.6. The categories b-QTOP and QT-SD are isomorphic.

Proof. Because of the previous propositions, it only remains to prove that the following statement is valid: For b-qtopological spaces $\left(X, \mathcal{B}^{X}, h_{1}\right)$ and $\left(Y, \mathcal{B}^{Y}, h_{2}\right)$, a bi-bounded function $f: X \longrightarrow Y$ is b-continuous if and only if it is a d-map between the subdensity spaces $\left(X, \mathcal{B}^{X}, N_{h_{1}}\right)$ and $\left(Y, \mathcal{B}^{Y}, N_{h_{2}}\right)$. By the assumption, $f$ is bi-bounded. Now, let $f$ be b-continuous. We have to verify that $f$ is a d-map. Let $B \in \mathcal{B}^{X} \backslash\{\emptyset\}$ and $\rho \in N_{h_{1}}(B)$. Hence, by the definition of $N_{h_{1}}$, we can find an element $x \in B$ with $x \in \bigcap\left\{h_{1}(F): F \in \rho \cap \mathcal{B}^{X}\right\}$. It remains to show that, for $A \in f \rho \cap \mathcal{B}^{Y}, f(x) \in h_{2}(A)$. Now, if $A \in f \rho \cap \mathcal{B}^{Y}$, then $A=f[F]$ for some $F \in \rho$ with $A \in \mathcal{B}^{Y}$. Hence, $f^{-1}[A]=f^{-1}[f[F]] \in \mathcal{B}^{X}$, and $F \in \rho \cap \mathcal{B}^{X}$ is valid. Consequently, $x \in h_{1}(F)$ and thus $f(x) \in h_{2}(f[F])=h_{2}(A)$.

Conversely, let $f$ be a d-map and $y \in f\left[h_{1}(B)\right]$ for $B \in \mathcal{B}^{X}$. We have to show that $y \in h_{2}(f[B])$. But $y=f(x)$ for some $x \in h_{1}(B)$, hence, $\{B\} \in N_{h_{1}}(\{x\})$ is valid. By the assumption, $\{f[B]\} \in N_{h_{2}}(\{f(x)\})$, which means that $y \in h_{2}(f[B])$. The proof is complete.

Proposition 3.7. For a b-qnear space $\left(X, \mathcal{B}^{X}, \xi\right)$ we set:

$$
N_{\xi}(\emptyset):=\{\emptyset\} \text { and } N_{\xi}(B):=\left\{\rho \in \underline{P} X:\{B\} \cup\left(\rho \cap \mathcal{B}^{X}\right) \in \xi\right\} \text {, if } B \neq \emptyset \text {. }
$$

Then, $\left(X, \mathcal{B}^{X}, N_{\xi}\right)$ is a subdensity space so that $c l_{N_{\xi}}^{b}=c l_{\xi}$, where $c l_{N_{\xi}}^{b}$ denotes the restriction of $c l_{N_{\xi}}$ to $\mathcal{B}^{X}$. 
Proof. $\quad\left(\operatorname{sd}_{1}\right): B \in \mathcal{B}^{X}$ implies $c l_{\xi}(B) \in \mathcal{B}^{X}$. If $x \in c l_{N_{\xi}}(B)$, then $\{B\} \in$ $N_{\xi}(\{x\})$ by the definition so that $\{\{x\}\} \cup\left(\{B\} \cap \mathcal{B}^{X}\right) \in \xi$. But $\{\{x\}, B\} \subseteq$ $\left\{\{x\} \cup\left(\{B\} \cap \mathcal{B}^{X}\right)\right.$, hence, $\{\{x\}, B\} \in \xi$ results, which yields $x \in \operatorname{cl}_{\xi}(B)$. Consequently, $c l_{N_{\xi}}(B) \in \mathcal{B}^{X}$ is valid, according to $\left(\mathrm{b}_{1}\right)$.

$\left(\operatorname{sd}_{2}\right):$ If $\rho \cap \mathcal{B}^{X} \in N_{\xi}(B), \rho \subseteq \underline{P} X$ and $B \in \mathcal{B}^{X} \backslash\{\emptyset\}$, then $\{B\} \cup\left(\left(\rho \cap \mathcal{B}^{X}\right) \cap \mathcal{B}^{X}\right) \in$ $\xi$ follows, so $\rho \in N_{\xi}(B)$.

$\left(\mathrm{sd}_{3}\right)$ : is evident.

$\left(\operatorname{sd}_{4}\right)$ : If $B \in \mathcal{B}^{X} \backslash\{\emptyset\}$, then $\{B\} \in N_{\xi}(B)$ because $\{B\}<<\{\{x\}\} \in \xi$ for some $x \in B$. Further, we have $\emptyset \in N_{\xi}(\emptyset)$. Now, suppose that $\mathcal{B}^{X} \in N_{\xi}(B)$ for some $B \in \mathcal{B}^{X} \backslash\{\emptyset\}$. Then, $\{\emptyset\} \in \xi$, which is a contradiction.

$\left(\mathrm{sd}_{5}\right)$ : is evident by the definition of $N_{\xi}$.

$\left(\mathrm{sd}_{6}\right)$ : Compare with $\left(\mathrm{sd}_{4}\right)$.

$\left(\mathrm{sd}_{7}\right)$ : is evident according to $\left(\mathrm{b}-\mathrm{n}_{3}\right)$.

$\left(\operatorname{sd}_{8}\right)$ : For $\rho \subseteq \underline{P} X$ and $B \in \mathcal{B}^{X} \backslash\{\emptyset\}$, let $\left\{c l_{N_{\xi}}(F): F \in \rho\right\} \in N_{\xi}(B)$. We have to verify that $\{B\} \cup\left(\rho \cap \mathcal{B}^{X}\right) \in \xi$. By the hypothesis, we get $\{B\} \cup$ $\left(\left\{c l_{N_{\xi}}(F): F \in \rho\right\} \cap \mathcal{B}^{X}\right) \in \xi$. Now, we will show that $\left\{c l_{\xi}(A): A \in\right.$ $\{B\} \cup\left(\rho \cap \mathcal{B}^{X}\right)<<\{B\} \cup\left(\left\{c_{N_{\xi}}(F): F \in \rho\right\} \cap \mathcal{B}^{X}\right)$. Namely, if this statement is true, then, $\left\{\operatorname{cl}_{\xi}(A): A \in\{B\} \cup\left(\rho \cap \mathcal{B}^{X}\right)\right\} \in \xi$ according to (b-n $\left.\mathrm{n}_{3}\right)$, and $\{B\} \cup\left(\rho \cap \mathcal{B}^{X}\right) \in \xi$ is valid by applying $\left(\mathrm{b}-\mathrm{n}_{6}\right)$. Let $c l_{\xi}(A)$ be chosen for $A \in \rho \cap \mathcal{B}^{X}$. Then, $c l_{N_{\xi}}(A) \in\left\{c l_{N_{\xi}}(F): F \in \rho\right\}$ and, moreover, $c l_{N_{\xi}}(A) \in \mathcal{B}^{X}$ because $c l_{N_{\xi}}(A) \subseteq \operatorname{cl}_{\xi}(A) \in \mathcal{B}^{X}$ (compare with the proof of $\left.\left(\operatorname{sd}_{1}\right)\right)$. Taking this into account, we get the desired result.

For each $B \in \mathcal{B}^{X}$, we have $c l_{N_{\xi}}^{b}(B) \subseteq c l_{\xi}(B)$. Conversely, let $B \in \mathcal{B}^{X}$ and $x \in c l_{\xi}(B)$. Then, $\{\{x\}, B\} \in \xi$, hence, $\{\{x\}\} \cup\left(\{B\} \cap \mathcal{B}^{X}\right) \in \xi$. Consequently, $x \in c l_{N_{\xi}}(B)=c l_{N_{\xi}}^{b}(B)$, which concludes the proof.

Remark 3.8. By the definition of $N_{\xi}$, the subdensity space $\left(X, \mathcal{B}^{X}, N_{\xi}\right)$ has an additional property, which we will take into account now.

Definition 3.9. Let us call a subdensity space $\left(X, \mathcal{B}^{X}, N\right)$ qnear iff $N$ possesses the following property:

(qn) $B \in \mathcal{B}^{X} \backslash\{\emptyset\}$ and $\rho \in N(B)$ imply $\{B\} \cup \rho \in \bigcap\left\{N(F): F \in\left(\rho \cap \mathcal{B}^{X}\right) \cup\right.$ $\{B\}\}$. We denote by QN-SD the full subcategory of SD whose objects are the qnear subdensity spaces.

Lemma 3.10. The subdensity space $\left(X, \mathcal{B}^{X}, N_{\xi}\right)$ is qnear.

Proof. For $B \in \mathcal{B}^{X} \backslash\{\emptyset\}$, let $\rho \in N_{\xi}(B)$. We have to verify that $\{B\} \cup \rho \in$ $\bigcap\left\{N_{\xi}(F): F \in\left(\rho \cap \mathcal{B}^{X}\right) \cup\{B\}\right\}$. Let $F \in\left(\rho \cap \mathcal{B}^{X}\right) \cup\{B\}$. We have to show that $\rho \in N_{\xi}(F)$. First, if $F=B$, we get $\{F\} \cup\left(\rho \cap \mathcal{B}^{X}\right) \in \xi$ by the hypothesis, hence, $\rho \in N_{\xi}(F)$. Second, if $F \in \rho \cap \mathcal{B}^{X}$, we have $\{F\} \cup\left(\rho \cap \mathcal{B}^{X}\right)<<\{B\} \cup\left(\rho \cap \mathcal{B}^{X}\right) \in \xi$. Consequently $\{F\} \cup\left(\rho \cap \mathcal{B}^{X}\right) \in \xi$, which results in $\rho \in N_{\xi}(F)$.

Proposition 3.11. Every qnear subdensity space $\left(X, \mathcal{B}^{X}, N\right)$ is closed, i.e., satisfying the following condition:

(clo) $B \in \mathcal{B}^{X}$ implies $N\left(\operatorname{cl}_{N}(B)\right)=N(B)$. 
Proof. If $\rho \in N\left(c_{N}(B)\right)$ for $B \in \mathcal{B}^{X}$, then, $\left\{c l_{N}(B)\right\} \cup \rho \in N\left(c_{N}(B)\right)$ according to (qn). Since $\left\{c l_{N}(A): A \in\{B\} \cup \rho\right\}<<\left\{c l_{N}(B)\right\} \cup \rho$ we get $\left\{c l_{N}(A)\right.$ : $A \in\{B\} \cup \rho\} \in N\left(c_{N}(B)\right)$ by applying $\left(\operatorname{sd}_{3}\right)$. Hence, $\{B\} \cup \rho \in N\left(c_{N}(B)\right)$ according to $\left(\operatorname{sd}_{8}\right)$. In the same way, we obtain $\left\{c l_{N}(B)\right\} \cup(\{B\} \cup \rho) \in N(B)$ with $\rho<<\left\{c l_{N}(B)\right\} \cup(\{B\} \cup \rho)$. Hence, by $\left(\operatorname{sd}_{3}\right)$, we get the desired result. The converse inclusion holds by $\left(\operatorname{sd}_{3}\right)$ and $\left(\operatorname{sd}_{7}\right)$.

Proposition 3.12. For a qnear subdensity space $\left(Y, \mathcal{B}^{Y}, M\right)$, the triple $\left(Y, \mathcal{B}^{Y}, \eta_{M}\right)$ is a b-qnear space such that $M=N_{\eta_{M}}$ where $\eta_{M}:=\left\{\mathcal{A} \in \underline{P} \mathcal{B}^{Y}\right.$ : $\mathcal{A} \in \bigcap\{M(A): A \in \mathcal{A}\}\}$.

Proof. $\left(\mathrm{b}-\mathrm{n}_{1}\right)$ : For $B \in \mathcal{B}^{X}$, we will show that the inclusion $\operatorname{cl}_{\eta_{M}}(B) \subseteq c l_{M}(B)$ is valid. $x \in c l_{\eta_{M}}(M)$ implies $\{\{x\}, B\} \in \eta_{M}$, hence, $\{\{x\}, B\} \in M(\{x\})$ and $\{B\}<<\{\{x\}, B\}$. Consequently, $\{B\} \in M(\{x\})$ follows, showing that $x \in \operatorname{cl}_{M}(B)$. By the hypothesis, $c l_{M}(B) \in B^{Y}$ is valid, and $c l_{\eta_{M}}(B) \in$ $\mathcal{B}^{Y}$ results.

(b-n $)$ : Now, let $\mathcal{A}_{2}<<\mathcal{A}_{1} \in \eta_{M}, \mathcal{A}_{2} \in \underline{P B^{Y}}$. For $A_{2} \in \mathcal{A}_{2}$, we can choose $A_{1} \in \mathcal{A}_{1}$ such that $A_{1} \subseteq A_{2}$. Hence, $\mathcal{A}_{1} \in M\left(A_{1}\right) \subseteq M\left(A_{2}\right)$ resulting in $\mathcal{A}_{2} \in M\left(A_{2}\right)$, and $\mathcal{A}_{2} \in \eta_{M}$ follows.

$\left(\mathrm{b}-\mathrm{n}_{3}\right)$ : is evident.

$\left(\mathrm{b}-\mathrm{n}_{4}\right)$ : is evident.

(b-n $):$ For $\mathcal{A} \in \underline{P} \mathcal{B}^{Y}$, let $\left\{c l_{\eta_{M}}(A): A \in \mathcal{A}\right\} \in \eta_{M}$. We have to verify that $\mathcal{A} \in \eta_{M}$. So, let $A \in \mathcal{A}$. We will show that $\mathcal{A} \in M(A)$. By the hypothesis, we get $\mathcal{A} \in M\left(c_{\eta_{M}}(A)\right) \subseteq M\left(c l_{M}(A)\right)=M(A)$. By Proposition 3.11, we obtain the desired result.

Finally, we have to verify that the equation $N_{\eta_{M}}=M$ is valid. Let $B \in \mathcal{B}^{X} \backslash\{\emptyset\}$. " $\leq$ " : $\rho \in N_{\eta_{M}}(B)$ implies $\{B\} \cup\left(\rho \cap \mathcal{B}^{X}\right) \in \eta_{M}$, hence, $\{B\} \cup\left(\rho \cap \mathcal{B}^{X}\right) \in M(B)$. Therefore, $\rho \cap \mathcal{B}^{X} \in M(B)$. But, then, $\rho \in M(B)$ according to $\left(\operatorname{sd}_{2}\right)$.

" $\geq ": \rho \in M(B)$ implies $\{B\} \cup \rho \in \bigcap\left\{M(F): F \in\left(\rho \cap \mathcal{B}^{X}\right) \cup\{B\}\right\}$ according to (qn). We have to show that $\{B\} \cup\left(\rho \cap \mathcal{B}^{X}\right) \in \eta_{M}$. So, let $F \in\{B\} \cup\left(\rho \cap \mathcal{B}^{X}\right)$. If $F=B$, then we have $\{B\} \cup \rho \in M(F)$ and, consequently, $\{B\} \cup\left(\rho \cap \mathcal{B}^{X}\right) \in M(F)$ by $\left(\operatorname{sd}_{3}\right)$. If $F \in \rho \cap \mathcal{B}^{X}$, we also have $\{B\} \cup \rho \in M(F)$ and, by the same argument as above, we get the desired result.

Corollary 3.13. For a qnear space $\left(X, \mathcal{B}^{X}, \xi\right)$ the equation $\eta_{N_{\xi}}=\xi$ is valid.

Proof. " $\leq$ ": $\mathcal{A} \in \eta_{N_{\xi}}$ implies $\mathcal{A} \in \bigcap\left\{N_{\xi}(F): F \in \mathcal{A}\right\}$. Let $\mathcal{A} \neq \emptyset$. Choose $A \in \mathcal{A}$, hence, $\mathcal{A} \in N_{\xi}(A)$. This implies $\{A\} \cup\left(\mathcal{A} \cap \mathcal{B}^{X}\right) \in \xi$. But $\mathcal{A} \cap \mathcal{B}^{X}=\mathcal{A}$, and, consequently, $\mathcal{A} \in \xi$ follows.

" $\geq$ ": Conversely, let $\mathcal{A} \in \xi$ and $F \in \mathcal{A}$. Then, $\{F\} \cup\left(\mathcal{A} \cap \mathcal{B}^{X}\right) \in \xi$ by the hypothesis and so $\mathcal{A} \in N_{\xi}(F)$, which concludes the proof.

Theorem 3.14. The categories $b-Q N E A R$ and $Q N-S D$ are isomorphic.

Proof. According to the previous statements, the above constructed connection is bijective and, moreover, a b-near map can be easily described by its corresponding d-map. 


\section{BOUNDED TOPOLOGY AND BOUNDED NEARNESS}

Now, we will fill the gap between b-quasitopologies and b-topologies and, analogously, that between b-quasinearness and b-nearness by introducing a further important property for subdensity spaces.

Definition 4.1. For a given subdensity space $\left(X, \mathcal{B}^{X}, N\right)$, we call $N$ bonded if it possesses the following property:

(bon) $B \in \mathcal{B}^{X}$ and $\rho_{1} \vee \rho_{2} \in N(B)$ imply $\rho_{1} \in N(B)$ or $\rho_{2} \in N(B)$.

Remark 4.2. Now, in this context, we also give two corresponding properties for b-qtopological and b-qnear spaces as follows:

Definition 4.3. A b-qtopological space $\left(X, \mathcal{B}^{X}, h\right)$ is called a b-topological space if $\mathcal{B}^{X}$ is bornology in the sense of [8] and $h$ satisfies the condition

(b-t $\left.\mathrm{t}_{6}\right) B_{1}, B_{2} \in \mathcal{B}^{X}$ imply $h\left(B_{1} \cup B_{2}\right) \subseteq h\left(B_{1}\right) \cup h\left(B_{2}\right)$.

We denote by b-TOP the full subcategory of b-QTOP whose objects are the btopological spaces.

Example 4.4. For a set $X$, let $\mathcal{B}_{f}^{X}$ denote the set of all finite subsets of $X$. Thus, $\mathcal{B}_{f}^{X}$ defines a bornology on $X$ : Here, a bornology $\mathcal{B}^{X}$ is subset of $\underline{P} X$ possessing the following properties:

$\left(b_{0}\right) \emptyset \in \mathcal{B}^{X}$

(b) $B_{2} \subset B_{1} \in \mathcal{B}^{X}$ imply $B_{2} \in \mathcal{B}^{X}$ :

$\left(b_{2}\right) B \subset X$ finite implies $B \in \mathcal{B}^{X}$;

(b) $B_{1}, B_{2} \in \mathcal{B}^{X}$ imply $B_{1} \cup B_{2} \in \mathcal{B}^{X}$.

Then, for a fixed element $x \in X$, we establish a b-topology $t^{x}: \mathcal{B}^{X} \longrightarrow \underline{P} X$ by setting:

$t^{x}(\emptyset):=\emptyset$ and

$t^{x}(B):=B \cup\{x\}$ if $B \neq \emptyset$.

Definition 4.5. Now, we call a qtopological subdensity space $\left(X, \mathcal{B}^{X}, N\right)$ a topological subdensity space if $\mathcal{B}^{X}$ is bornology and $N$ is bonded. We denote by T-SD the full subcategory of QT-SD whose objects are the topological subdensity spaces.

Theorem 4.6. The categories $b$-TOP and T-SD are isomorphic.

Proof. By Theorem 3.6, it only remains to show that $N_{h}$ is satisfying the condition (bon). From $B \in \mathcal{B}^{X} \backslash\{\emptyset\}$ and $\rho_{1} \vee \rho_{2} \in N_{h}(B)$ it follows that there is an element $x \in B$ such that $x \in \bigcap\left\{h(F): F \in\left(\rho_{1} \vee \rho_{2}\right) \cap \mathcal{B}^{X}\right\}$. Assuming $\rho_{1}, \rho_{2} \notin N_{h}(B)$, we can find a set $F_{1} \in \rho_{1} \cap \mathcal{B}^{X}$ and a set $F_{2} \in \rho_{2} \cap \mathcal{B}^{X}$ such that $x \notin F_{1} \cup F_{2}$. But $F_{1} \cup F_{2} \in\left(\rho_{1} \vee \rho_{2}\right) \cap \mathcal{B}^{X}$ is valid, hence, $x \in F_{1} \cup F_{2}$, which is a contradiction.

Remark 4.7. Here, we point out that above considered spaces are special cases of the so-called supernear spaces in the sense of [12]. But, in that general context, only the usual topological spaces (given by the Kuratowski closure operators) can be considered as particular saturated supernear spaces, in which the set $X$ is an element of the given boundedness $\mathcal{B}^{X}$. But here, we obtain a better result, because 
even arbitrary b-topological spaces are now involved in this new theory presented. Moreover, we will show that this fact can analogously be extended to the so-called b-nearness spaces.

Definition 4.8. We call a b-qnear space $\left(X, \mathcal{B}^{X}, \xi\right)$ a b-nearness space or shortly a $b$-near space if $\mathcal{B}^{X}$ is a bornology and $\xi$ satisfies the condition (b-n $\left.\mathrm{n}_{6}\right) \rho_{1}, \rho_{2} \in \underline{P \mathcal{B}^{X}}$ and $\rho_{1} \vee \rho_{2} \in \xi$ imply $\rho_{1} \in \xi$ or $\rho_{2} \in \xi$.

We denote by b-NEAR the full subcategory of b-QNEAR whose objects are the b-near spaces.

Remark 4.9. If we consider a symmetric b-topological space $\left(X, \mathcal{B}^{X}, t\right)$, then the triple $\left(X, \mathcal{B}^{X}, \xi_{t}\right)$, where $\xi_{t}:=\left\{\rho \in \underline{P} \mathcal{B}^{X}: \bigcap\{t(F): F \in \rho\} \neq \emptyset\right\}$, defines a b-near space. Note that, in this case, we have $c l_{\xi_{t}}=t$. Recall that each btopological space $\left(X, \mathcal{B}^{X}, t\right)$ induces a symmetric b-topological space $\left(X, \mathcal{B}^{X}, t^{s}\right)$ where $t^{s}(B):=\{z \in X: \exists x \in B(x \in t(\{z\})$ and $z \in t(\{x\})\}$.

Definition 4.10. We call a qnear subdensity space $\left(X, \mathcal{B}^{X}, N\right)$ a near subdensity space if $\mathcal{B}^{X}$ is a bornology and $N$ is bonded. We denote by N-SD the full subcategory of QN-SD whose objects are the near subdensity spaces. If $\rho \in N(B)$ for some $B \in \mathcal{B}^{X}$, then we call $\rho$ a $B$-near collection in $N$.

Theorem 4.11. The categories $b-N E A R$ and $N-S D$ are isomorphic.

Proof. By Theorem 3.14, it only remains to verify that $N_{\xi}$ is bonded. Let $\rho_{1} \vee \rho_{2} \in N_{\xi}(B), B \in \mathcal{B}^{X}, \rho_{1}, \rho_{2} \subseteq \underline{P} X$. Then, $\{B\} \cup\left(\left(\rho_{1} \vee \rho_{2}\right) \cap \mathcal{B}^{X}\right) \in \xi$. But $\{B\} \cup\left(\rho_{1} \cap \mathcal{B}^{X}\right) \vee\{B\} \cup\left(\rho_{2} \cap \mathcal{B}^{X}\right)<<\{B\} \cup\left(\left(\rho_{1} \vee \rho_{2}\right) \cap \mathcal{B}^{X}\right)$ and, according to (b-n $\left.\mathrm{n}_{6}\right)$, we get $\{B\} \cup\left(\rho_{1} \cap \mathcal{B}^{X}\right) \in \xi$ or $\{B\} \cup\left(\rho_{2} \cap \mathcal{B}^{X}\right) \in \xi$ showing that $\rho_{1} \in N_{\xi}(B)$ or $\rho_{2} \in N_{\xi}(B)$.

Conversely, let $\mathcal{A}_{1} \vee \mathcal{A}_{2} \in \eta_{M}, \mathcal{A}_{1}, \mathcal{A}_{2} \in \underline{P} \mathcal{B}^{X}$ and suppose $\mathcal{A}_{1}, \mathcal{A}_{2} \notin \eta_{M}$. Then, we can find $A_{1} \in \mathcal{A}_{1}$ with $\mathcal{A}_{1} \notin M\left(A_{1}\right)$ and $A_{2} \in \mathcal{A}_{2}$ with $\mathcal{A}_{2} \notin M\left(A_{2}\right)$. But $A_{1} \cup A_{2} \in \mathcal{A}_{1} \vee \mathcal{A}_{2}$ where $\mathcal{A}_{1} \vee \mathcal{A}_{2} \in M\left(A_{1} \cup A_{2}\right)$ by the hypothesis. Since $M$ is bonded, we obtain $\mathcal{A}_{1} \in M\left(A_{1} \cup A_{2}\right)$ or $\mathcal{A}_{2} \in M\left(A_{1} \cup A_{2}\right)$. In the first case, we get $\left\{A_{1} \cup A_{2}\right\} \cup \mathcal{A}_{1} \in M\left(A_{1}\right)$ by (qn) so that $\mathcal{A}_{1} \in M\left(A_{1}\right)$, which is a contradiction. The second case is analogous.

Remark 4.12. The spaces considered above are special cases of the so-called paranear spaces in the sense of [15]. But, in that general context, only the wellknown nearness spaces can be considered as saturated paranear spaces. So, we again obtain a better result because now even arbitrary b-nearness spaces are being involved in this new theory presented.

\section{TOPOlOGicAl EXtensions AND RELATED SD-SPACES}

We will now deal with the problem of finding a one-to-one correspondence between certain topological extensions and their related sd-spaces. First, we define the category BTEXT of the so-called bornotopological extensions - shortly btop-extensions - and related morphisms as follows:

Definition 5.1. The objects of BTEXT are triples $E:=\left(e, \mathcal{B}^{X}, Y\right)$ where $X:=\left(X, t_{X}\right)$ and $Y:=\left(Y, t_{Y}\right)$ are topological spaces (given by closure operators $t_{X}$ 
and $t_{Y}$, respectively), $\mathcal{B}^{X}$ is a bornology such that $B \in \mathcal{B}^{X}$ implies $t_{X}(B) \in \mathcal{B}^{X}$, and

$e: X \longrightarrow Y$ is a function satisfying the following conditions:

$\left(\right.$ btx $\left._{1}\right) B \in \mathcal{B}^{X}$ implies $t_{X}(B)=e^{-1}\left[t_{Y}(e[B])\right]$, where $e^{-1}$ denotes the inverse image under $e$.

$\left(\right.$ btx $\left._{2}\right) t_{Y}(e[X])=Y$, which means that the image of $X$ under $e$ is dense in $Y$.

The morphisms in BTEXT are of the form $(f, g):\left(e, \mathcal{B}^{X}, Y\right) \longrightarrow\left(e^{\prime}, \mathcal{B}^{X^{\prime}}, Y^{\prime}\right)$ where $f: X \longrightarrow X^{\prime}$ and $g: Y \longrightarrow Y^{\prime}$ are continuous maps such that $f$ is bibounded, and the following diagram commutes

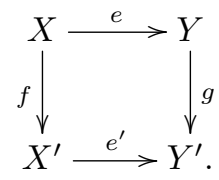

If $(f, g):\left(e, \mathcal{B}^{X}, Y\right) \longrightarrow\left(e^{\prime}, \mathcal{B}^{X^{\prime}}, Y^{\prime}\right)$ and $\left(f^{\prime}, g^{\prime}\right):\left(e^{\prime}, \mathcal{B}^{X^{\prime}}, Y^{\prime}\right) \longrightarrow\left(e^{\prime \prime}, \mathcal{B}^{X^{\prime \prime}}, Y^{\prime \prime}\right)$ are BTEXT-morphisms, then they can be composed according to the rule $\left(f^{\prime}, g^{\prime}\right) \circ$ $(f, g):=\left(f^{\prime} \circ f, g^{\prime} \circ g\right):\left(e, \mathcal{B}^{X}, Y\right) \longrightarrow\left(e^{\prime \prime}, \mathcal{B}^{X^{\prime \prime}}, Y^{\prime \prime}\right)$, where "०" denotes the composition of maps.

Remark 5.2. Observe that the axiom $\left(\mathrm{btx}_{1}\right)$ in Definition 5.1 is automatically satisfied if $e: X \longrightarrow Y$ is a topological embedding. Moreover, we consider an ordinary bornology $\mathcal{B}^{X}$, which need not coincide with the power set $\underline{P} X$.

Definition 5.3. We call a btop-extension $E:=\left(e, \mathcal{B}^{X}, Y\right)$

(i) strict $[1]$ if it satisfies

(str) $\left\{t_{Y}(e[A]): A \subseteq X\right\}$ forms a base for the closed subsets of $Y$;

(ii) symmetric $[3]$ if it satisfies

(sy) $x \in X$ and $y \in t_{Y}(\{e(x)\})$ imply $e(x) \in t_{Y}(\{y\})$.

Examples 5.4. (i) For a bornotopological extension $E:=\left(e, \mathcal{B}^{X}, Y\right)$ we consider the triple $\left(X, \mathcal{B}^{X}, N_{e}\right)$, where $N_{e}$ is defined by setting:

$N_{e}(\emptyset):=\{\emptyset\}$ and

$N_{e}(B):=\{\rho \subseteq \underline{P} X: \exists y \in e[B]\} y \in \bigcap\left\{t_{Y}(e[F]): F \in \rho \cap \mathcal{B}^{X}\right\}$ if $B \neq \emptyset$.

(ii) For a symmetric bornotopological extension $E:=\left(e, \mathcal{B}^{X}, Y\right)$ we consider the triple $\left(X, \mathcal{B}^{X}, N^{e}\right)$ where $N^{e}$ is defined by setting:

$N^{e}(\emptyset):=\{\emptyset\}$ and

$N^{e}(B):=\left\{\rho \subseteq \underline{P} X: \bigcap\left\{t_{Y}(e[F]): F \in\left(\rho \cap \mathcal{B}^{X}\right) \cup\{B\}\right) \neq \emptyset\right\}$ if $B \neq \emptyset$.

Lemma 5.5. The triple $\left(X, \mathcal{B}^{X}, N^{e}\right)$ is a near sd-space such that $c_{N^{e}}(B)=$ $t_{X}(B)$ for each $B \in \mathcal{B}^{X}$.

Proof. First, we will verify that $\operatorname{cl}_{N^{e}}(B)=t_{X}(B)$ for each $B \in \mathcal{B}^{X}$. To this end, let $B \in \mathcal{B}^{X} \backslash\{\emptyset\}$.

"ᄃ": $x \in \operatorname{cl}_{N^{e}}(B)$ implies $\{B\} \in N^{e}(\{x\})$. Hence, we can find $y \in t_{Y}(\{e(x)\})$ with $y \in t_{Y}(e[B])$. By (sy), we get $e(x) \in t_{Y}(\{y\})$, and $e(x) \in t_{Y}(e[B])$ follows because $t_{Y}$ is topological . Consequently, $x \in e^{-1}\left[t_{Y}(e[B])\right]$ is valid, which shows that $x \in t_{X}(B)$ by $\left(\mathrm{btx}_{1}\right)$. 


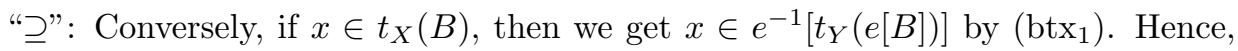
$e(x) \in t_{Y}(e[B])$ and, consequently, $\{B\} \in N^{e}(\{x\})$. Therefore, $x \in c l_{N^{e}}(B)$.

Now, $\left(X, \mathcal{B}^{X}, N^{e}\right)$ evidently satisfies $\left(\operatorname{sd}_{1}\right)$, since $t_{X}(B) \in \mathcal{B}^{X}$ for each $B \in \mathcal{B}^{X}$ according to Definition 5.1.

$\left(\operatorname{sd}_{2}\right)$ : For $B \in \mathcal{B}^{X} \backslash\{\emptyset\}$ and $\rho \subseteq \underline{P} X$, Let $\rho \cap \mathcal{B}^{X} \in N^{e}(B)$. Then, there exists an element $y \in t_{Y}(e[B])$ such that $y \in \bigcap\left\{t_{Y}(e[F]): F \in\left(\rho \cap \mathcal{B}^{X}\right) \cap \mathcal{B}^{X}\right\}$. But $\left(\rho \cap \mathcal{B}^{X}\right) \cap \mathcal{B}^{X}=\rho \cap \mathcal{B}^{X}$, and $\rho \in N^{e}(B)$ follows.

$\left(\operatorname{sd}_{3}\right): \rho_{2}<<\rho_{1} \in N^{e}(B), \rho_{2} \subseteq \underline{P} X$ and $B \in \mathcal{B}^{X} \backslash\{\emptyset\}$ imply the existence of $y \in t_{Y}(e[F])$ with $y \in \bigcap\left\{t_{Y}\left(e\left[F_{1}\right]\right): F_{1} \in \rho_{1} \cap \mathcal{B}^{X}\right\}$. Now, let $F_{2} \in \rho_{2} \cap \mathcal{B}^{X}$. Then, we can choose $F_{1}^{\prime} \in \rho$ such that $F_{1}^{\prime} \subseteq F_{2}$. It follows that $F_{1}^{\prime} \in \mathcal{B}^{X}$ by applying (b $\left.b_{1}\right)$. Hence, $y \in t_{Y}\left(e\left[F_{1}\right]\right) \subseteq t_{Y}\left(e\left[F_{2}\right]\right)$ results so that $\rho_{2} \in N^{e}(B)$ is valid.

$\left(\operatorname{sd}_{4}\right)$ : For $B \in \mathcal{B}^{X}$, suppose that $\mathcal{B}^{X} \in N^{e}(B)$. In the case $B=\emptyset$, this contradicts the definition of $N^{e}$. In the case $B \neq \emptyset$, there exists $y \in t_{Y}(e[B])$ such that $y \in \bigcap\left\{t_{Y}(e[F]): F \in \mathcal{B}^{X}\right\}$. But then, $y \in t_{Y}(\emptyset)=\emptyset$ follows, which is also a contradiction. We have $\emptyset \in N^{e}(\emptyset)$ and, if $B \in \mathcal{B}^{X} \backslash\{\emptyset\}$, we can choose $x \in B$. Hence, $e(x) \in t_{Y}(e[B])$ follows and $\{B\} \in N^{e}(B)$ results. Consequently, the axiom $\left(\mathrm{sd}_{6}\right)$ is also fulfilled. By the definition of $N^{e},\left(\mathrm{sd}_{5}\right)$ is valid.

$\left(\operatorname{sd}_{7}\right)$ : If $\emptyset \neq B_{2} \subseteq B_{1} \in \mathcal{B}^{X}$ and $\rho \in N^{e}\left(B_{2}\right), \rho \subseteq \underline{P} X$, then we can choose $y \in t_{Y}\left(e\left[B_{2}\right]\right)$ with $y \in \bigcap\left\{t_{Y}(e[F]): F \in \rho \cap \mathcal{B}^{X}\right\}$. But $t_{Y}\left(e\left[B_{2}\right]\right) \subseteq t_{Y}\left(e\left[B_{1}\right]\right)$ results in $\rho \in N^{e}\left(B_{1}\right)$.

$\left(\operatorname{sd}_{8}\right)$ : Let $\left\{c l_{N^{e}}(F): F \in \rho\right\} \in N^{e}(B), \rho \subseteq \underline{P} X$ and $B \in \mathcal{B}^{X} \backslash\{\emptyset\}$. Then, we can find an element $y \in t_{Y}(e[B])$ such that $y \in \bigcap\left\{t_{Y}(e[A]): A \in\left\{c_{N^{e}}(F): F \in\right.\right.$ $\left.\rho\} \cap \mathcal{B}^{X}\right\}$. Now, if $F^{\prime} \in \rho \cap \mathcal{B}^{X}$, then $c_{N^{e}}\left(F^{\prime}\right) \in\left\{c l_{N^{e}}(F): F \in \rho\right\}$. Moreover, we have $c l_{N^{e}}\left(F^{\prime}\right) \in \mathcal{B}^{X}$. Hence, $y \in t_{Y}\left(e\left[c l_{N^{e}}\left(F^{\prime}\right)\right]\right)$. Since $c l_{N^{e}}\left(F^{\prime}\right)=t_{X}\left(F^{\prime}\right)$, we get $y \in t_{Y}\left(e\left[t_{X}\left(F^{\prime}\right)\right]\right) \subseteq t_{Y}\left(t_{Y}\left(e\left[F^{\prime}\right]\right)\right) \subseteq t_{Y}\left(e\left[F^{\prime}\right]\right)$ because $t_{Y}$ is topological. Consequently, $\rho \in N^{e}(B)$.

(qn): For $B \in \mathcal{B}^{X} \backslash\{\emptyset\}$, let $\rho \in N^{e}(B)$. We have to show that $\{B\} \cup \rho \in$ $\bigcap\left\{N^{e}(F): F \in\left(\rho \cap \mathcal{B}^{X}\right) \cup\{B\}\right\}$. To this end, let $\rho \in N^{e}(B)$. Then, there exists $y \in t_{Y}(e[B])$ such that $y \in \bigcap\left\{t_{Y}(e[F]): F \in \rho \cap \mathcal{B}^{X}\right\}$. We have $\{B\} \cup \rho \in N^{e}(B)$ because, for $A \in(\{B\} \cup \rho) \cap \mathcal{B}^{X}, y \in t_{Y}(e[A])$ by the hypothesis. On the other hand, if $A \in(\{B\} \cup \rho) \cap \mathcal{B}^{X}$, then we also have $\{B\} \cup \rho \in N^{e}(A)$. Therefore, (qn) is valid.

We have shown that $\left(X, \mathcal{B}^{X}, N^{e}\right)$ is a qnear sd-space. But then, $\left(X, \mathcal{B}^{X}, N^{e}\right)$ is a near sd-space because $\mathcal{B}^{X}$ is a bornology and $N^{e}$ is bonded. This can be verified as follows: For $\rho_{1}, \rho_{2} \subseteq \underline{P} X$, let $\rho_{1} \vee \rho_{2} \in N^{e}(B)$ and $B \in \mathcal{B}^{X} \backslash\{\emptyset\}$. Suppose that $\rho_{1}, \rho_{2} \notin N^{e}(B)$. By the hypothesis, we can find $y \in t_{Y}(e[B])$ with $y \in \bigcap\left\{t_{Y}(e[F]): F \in \rho \cap \mathcal{B}^{X}\right\}$. On the other hand, there exists $F_{1} \in \rho_{1} \cap \mathcal{B}^{X}$ with $y \notin t_{Y}\left(e\left[F_{1}\right]\right)$ and $F_{2} \in \rho_{2} \cap \mathcal{B}^{X}$ with $y \notin t_{Y}\left(e\left[F_{2}\right]\right)$. Hence, $F:=F_{1} \cup F_{2} \in$ $\left(\rho_{1} \vee \rho_{2}\right) \cap \mathcal{B}^{X}$ because $\mathcal{B}^{X}$ is a bornology. Consequently, $y \in t_{Y}(e[F])$. But $t_{Y}(e[F])=t_{Y}\left(e\left[F_{1} \cup F_{2}\right]\right)=t_{Y}\left(e\left[F_{1}\right]\right) \cup t_{Y}\left(e\left[F_{2}\right]\right)$, hence, $y \in t_{Y}\left(e\left[F_{1}\right]\right) \cup t_{Y}\left(e\left[F_{2}\right]\right)$, which is a contradiction.

Definition 5.6. For a subdensity space $\left(X, \mathcal{B}^{X}, N\right)$, let $B \in \mathcal{B}^{X} \backslash\{\emptyset\}$. A subset $\mathcal{C} \subseteq \underline{P} X$ is called a $B$-clan in $N$ if it satisfies the following conditions:

$\left(\right.$ bcla $\left._{1}\right) \emptyset \notin \mathcal{C}$;

(bcla 2$) C_{1} \in \mathcal{C}$ and $C_{1} \subseteq C_{2} \in \underline{P} X$ imply $C_{2} \in \mathcal{C}$;

$\left(\right.$ bcla $\left._{3}\right) C_{1} \cup C_{2} \in \mathcal{C}$ implies $C_{1} \in \mathcal{C}$ or $C_{2} \in \mathcal{C}$; 
$\left(\mathrm{bcla}_{4}\right) \quad B \in \mathcal{C} \in N(B)$;

(bcla 5$) \quad A \in \mathcal{C}$ and $A \subseteq c_{N}(F), F \in \mathcal{B}^{X}$ imply $F \in \mathcal{C}$.

Remark 5.7. For a subdensity space $\left(X, \mathcal{B}^{X}, N\right)$ and each $B \in \mathcal{B}^{X}$ with $x \in B$, $x_{N}:=\left\{A \subseteq X: x \in c l_{N}(A)\right\}$ is a B-clan in $N$. Moreover, we note that $x_{N}$ is a maximal element in $N(\{x\}) \backslash\{\emptyset\}$ with respect to set inclusion.

Definition 5.8. A subdensity space $\left(X, \mathcal{B}^{X}, N\right)$ is called a clan space if it satisfies the following condition:

(cla) $B \in \mathcal{B}^{X} \backslash\{\emptyset\}$ and $\rho \in N(B)$ imply the existence of a B-clan $\mathcal{C}$ in $N$ with $\rho \cap \mathcal{B}^{X} \subseteq \mathcal{C}$.

Remark 5.9. Every topological subdensity space $\left(X, \mathcal{B}^{X}, N\right)$ is a clan space, which can be shown as follows: For $B \in \mathcal{B}^{X} \backslash\{\emptyset\}$, let $\rho \in N(B)$. Then, there exists an element $x \in B$ with $x \in \bigcap\left\{c l_{N}(F): F \in \rho \cap \mathcal{B}^{X}\right\}$. We will show that the inclusion $\rho \cap \mathcal{B}^{X} \subseteq x_{N}$ is valid. But $F \in \rho \cap \mathcal{B}^{X}$ implies $x \in c l_{N}(F)$, hence, $F \in x_{N}$. By Remark 5.7, $x_{N}$ is a B-clan in $N$. Further, we state that the near subdensity space $\left(X, \mathcal{B}^{X}, N^{e}\right)$ is a clan space, too. In fact, given $B \in \mathcal{B}^{X} \backslash\{\emptyset\}$ and $\rho \in N^{e}(B)$, there exists $y \in t_{Y}(e[B])$ such that $y \in \bigcap\left\{t_{Y}(e[F]): F \in \rho \cap \mathcal{B}^{X}\right\}$. Now, we set $\mathcal{C}_{y}:=\left\{C \subseteq X: y \in t_{Y}(e[C])\right\}$. Evidently, $\rho \cap \mathcal{B}^{X} \subseteq \mathcal{C}_{y}$ is valid. It remains to verify that $\mathcal{C}_{y}$ satisfies the axioms $\left(\right.$ bcla $\left._{1}\right)$ to $\left(\right.$ bcla $\left._{5}\right)$ for being a B-clan in $N^{e}$.

$\left(\right.$ bcla $\left._{1}\right)$ : is evident.

$($ bcla $): C_{1} \in \mathcal{C}_{y}$ and $C_{1} \subseteq C_{2} \in \underline{P} X$ imply $y \in t_{Y}\left(e\left[C_{1}\right]\right) \subseteq t_{Y}\left(e\left[C_{2}\right]\right)$, hence, $C_{2} \in \mathcal{C}_{y}$ is valid.

$\left(\right.$ bcla $\left._{3}\right): C_{1} \cup C_{2} \in \mathcal{C}_{y}$ implies $y \in t_{Y}\left(e\left[C_{1} \cup C_{2}\right]\right)$. But $t_{Y}\left(e\left[C_{1} \cup C_{2}\right]\right)=t_{Y}\left(e\left[C_{1}\right]\right) \cup$ $t_{Y}\left(e\left[C_{2}\right]\right)$ and the statement $C_{1} \in \mathcal{C}_{y}$ or $C_{2} \in \mathcal{C}_{y}$ is valid.

$\left(\right.$ bcla $\left._{4}\right): \mathcal{C}_{y} \in N^{e}(B)$ because $y \in t_{Y}(e[B])$ and $F \in \mathcal{C}_{y} \cap \mathcal{B}^{X}$ implies $y \in t_{Y}(e[F])$. So, we have $y \in \bigcap\left\{t_{Y}(e[A]): A \in\left(\mathcal{C}_{y} \cap \mathcal{B}^{X}\right) \cup\{B\}\right\}$. Thus, $\mathcal{C}_{y} \in N^{e}(B)$.

$\left(\right.$ bcla $\left._{5}\right): A \in \mathcal{C}_{y}, A \subseteq c_{N^{e}}(F)$ and $F \in \mathcal{B}^{X}$ imply $y \in t_{Y}(e[A])$. But $t_{Y}(e[A]) \subseteq$ $t_{Y}\left(e\left[c l_{N^{e}}(F)\right]\right)=t_{Y}\left(e\left[t_{X}[F]\right]\right)$ according to Lemma 5.5.

By $\left(\operatorname{btx}_{1}\right)$, we get $t_{Y}\left(e\left[t_{X}(F)\right]\right) \subseteq t_{Y}\left(t_{Y}(e[F])\right) \subseteq t_{Y}(e[F])$, since $t_{Y}$ is topological. Taking this into account, we obtain $y \in t_{Y}(e[F])$, hence, $F \in \mathcal{C}_{y}$ is valid, which concludes the proof.

Convention 5.10. We denote by SYBTEXT the full subcategory of BTEXT whose objects are the symmetric btop-extensions and by NCLA-SD the full subcategory of SD whose objects are the near clan spaces.

Theorem 5.11. Let $H: S Y B T E X T \longrightarrow N C L A-S D$ be defined by

(a) For a SYBTEXT-object $E:=\left(e, \mathcal{B}^{X}, Y\right)$ we put $H(E):=\left(X, \mathcal{B}^{X}, N^{e}\right)$;

(b) for a BTEXT-morphism $(f, g): E \longrightarrow E^{\prime}$ we put $H(f, g):=f$.

Then, $H: S Y B T E X T \longrightarrow N C L A-S D$ is a functor.

Proof. We already know that the image of $H$ lies in NCLA-SD. Now, let $(f, g)$ : $\left(e, \mathcal{B}^{X}, Y\right) \longrightarrow\left(e^{\prime}, \mathcal{B}^{X^{\prime}}, Y^{\prime}\right)$ be a BTEXT-morphism. It has to be shown that $f$ is a d-map. By the hypothesis, $f$ is bi-bounded. Let $B \in \mathcal{B}^{X} \backslash\{\emptyset\}$ and $\rho \in$ $N^{e}(B)$. We have to verify that $f \rho \in N^{e^{\prime}}(f[B])$. By the hypothesis, we can choose $y \in t_{Y}(e[B])$ with $y \in \bigcap\left\{t_{Y}(e[F]): F \in \rho \cap \mathcal{B}^{X}\right\}$. We put $y^{\prime}:=g(y)$, hence, 
$y^{\prime} \in t_{Y^{\prime}}(f[B])$ because $g(y) \in g\left(t_{Y}(e[B])\right) \subseteq t_{Y^{\prime}}(g[e[B]])$ as $g$ is continuous. By the hypothesis, the corresponding diagram commutes. Hence, $g[e[B]]=e^{\prime}[f[B]]$, and thus $t_{Y^{\prime}}(g[e[B]])=t_{Y^{\prime}}\left(e^{\prime}[f[B]]\right)$. Consequently, $y \in t_{Y^{\prime}}\left(e^{\prime}[f[B]]\right)$ is valid. On the other hand, let $A \in f \rho \cap \mathcal{B}^{X^{\prime}}$ be an element. We have to verify that $y^{\prime} \in t_{Y^{\prime}}\left(e^{\prime}[A]\right)$.

Suppose $A=f[F]$ for some $F \in \rho$. Since $A \in \mathcal{B}^{X^{\prime}}$, we have $f^{-1}[A]=f^{-1}[f[F]] \supseteq$ $F \in \mathcal{B}^{X}$ because $f$ is rebounded and $\left(b_{1}\right)$ holds. Hence, $y \in t_{Y}(e[F])$ can be deduced from the hypothesis. But then, $y^{\prime}=g(y) \in g\left(t_{Y}(e[F])\right) \subseteq t_{Y^{\prime}}(g[e[F]])$ because $g$ is continuous, and $t_{Y^{\prime}}(g[e[F]])=t_{Y^{\prime}}\left(e^{\prime}[f[F]]\right)=t_{Y^{\prime}}\left(e^{\prime}[A]\right)$ by the commutativity of the corresponding diagram. Consequently, $y^{\prime} \in t_{Y^{\prime}}(e[A])$ is valid, which concludes the proof. Evidently, $H$ fulfills the remaining properties for being a functor.

\section{Strict BornotopologicAl EXtensions}

In the previous section, we have found a functor $H$ from SYBTEXT to NCLA-SD. Now, we are going to introduce a related one in the opposite direction.

Lemma 6.1. Let $\left(X, \mathcal{B}^{X}, N\right)$ be a near sd-space. We set $X^{C}:=\{\mathcal{C} \subseteq \underline{P} X: \mathcal{C}$ is a B-clan in $N$ for some $\left.B \in \mathcal{B}^{X} \backslash\{\emptyset\}\right\}$ and, for each $A^{C} \subseteq X^{C}$, we put: $t_{X^{C}}\left(A^{C}\right):=\left\{\mathcal{C} \in X^{C}: \triangle A^{C} \subseteq \mathcal{C}\right\}$ where $\triangle A^{C}:=\left\{F \in \mathcal{B}^{X}: \forall \mathcal{C} \in A^{C} F \in \mathcal{C}\right\}$. (By convention, $\triangle A^{C}=\mathcal{B}^{X}$ if $A^{C}=\emptyset$ ). Then, $t_{X^{C}}: \underline{P} X^{C} \longrightarrow \underline{P} X^{C}$ is a topological closure operator.

Proof. First, we note that $t_{X^{C}}(\emptyset)=\emptyset$ because $\emptyset \notin \mathcal{C}$ for each $\mathcal{C} \in X^{C}$. Let $A^{C}$ be a subset of $X^{C}$ and consider $\mathcal{C} \in A^{C}$. Then, $F \in \triangle A^{C}$ implies $F \in \mathcal{C}$, hence, $A^{C} \subseteq t_{X^{C}}\left(A^{C}\right)$ is valid. If $A_{1}^{C} \subseteq A_{2}^{C}$, then $\triangle A_{2}^{C} \subseteq \triangle A_{1}^{C}$ implying $t_{X^{C}}\left(A_{1}^{C}\right) \subseteq$ $t_{X^{C}}\left(A_{2}^{C}\right)$. For arbitrary subsets $A_{1}^{C}, A_{2}^{C} \subseteq X^{C}$, we consider an element $\mathcal{C} \in X^{C}$ such that $\mathcal{C} \notin t_{X^{C}}\left(A_{1}^{C}\right) \cup t_{X^{C}}\left(A_{2}^{C}\right)$. Then, we get $\triangle A_{1}^{C} \nsubseteq \mathcal{C}$ and $\triangle A_{2}^{C} \nsubseteq \mathcal{C}$. We can choose $F_{1} \in \triangle A_{1}^{C}$ with $F_{1} \notin \mathcal{C}$ and $F_{2} \in \triangle A_{2}$ with $F_{2} \notin \mathcal{C}$. By (bcla 3 ), we get $F_{1} \cup F_{2} \notin \mathcal{C}$. On the other hand, $F_{1} \cup F_{2} \in \mathcal{B}^{X}$, and $F_{1} \cup F_{2} \in \triangle A_{1}^{C} \cap$ $\triangle A_{2}^{C} \subseteq \triangle\left(A_{1}^{C} \cup A_{2}^{C}\right)$ imply $\mathcal{C} \notin t_{X^{C}}\left(A_{1}^{C} \cup A_{2}^{C}\right)$. Finally, let $\mathcal{C}$ be an element of $t_{X^{C}}\left(t_{X^{C}}\left(A^{C}\right)\right), A^{C} \subseteq X^{C}$, and suppose $\mathcal{C} \notin t_{X^{C}}\left(A^{C}\right)$. We can choose $F \in \triangle A^{C}$ with $F \notin \mathcal{C}$. By the assumption, we have $\triangle t_{X^{C}}\left(A^{C}\right) \subseteq \mathcal{C}$, hence, $F \notin \triangle t_{X^{C}}\left(A^{C}\right)$. Consequently, there exists $\mathcal{D} \in t_{X^{C}}\left(A^{C}\right)$ with $F \notin \mathcal{D}$. But this implies $\triangle A^{C} \subseteq \mathcal{D}$, and $F \in \mathcal{D}$ results, which is a contradiction.

Theorem 6.2. For near sd-spaces $\left(X, \mathcal{B}^{X}, N\right),\left(Y, \mathcal{B}^{Y}, M\right)$, let $f: X \longrightarrow Y$ be a d-map. Define a function $f^{C}: X^{C} \longrightarrow Y^{C}$ by setting $f^{C}(\mathcal{C}):=\{D \subseteq Y$ : $\left.f^{-1}\left[c l_{M}(D)\right] \in \mathcal{C}\right\}$ for each $\mathcal{C} \in X^{C}$. Then, the following statements are valid:

(1) $f^{C}$ is a continuous map from $\left(X^{C}, t_{X^{C}}\right)$ to $\left(Y^{C}, t_{Y^{C}}\right)$;

(2) The composites $f^{C} \circ e_{X}$ and $e_{Y} \circ f$ coincide where $e_{X}: X \longrightarrow X^{C}$ denotes that function which assigns the $\{x\}$-clan $x_{N}$ to each $x \in X$.

Proof. First, let $\mathcal{C}$ be a B-clan in $N$. We will show that $f^{C}(\mathcal{C})$ is a $f[B]$-clan in $M$. It is easy to verify that $f^{C}(\mathcal{C})$ satisfies the conditions $\left(\right.$ bcla $\left._{1}\right)$ to $\left(\right.$ bcla $\left._{3}\right)$ in Definition 5.6. In order to prove $\left(\right.$ bcla $\left._{4}\right)$, we observe that $B \in \mathcal{C} \in N(B)$ is valid by the hypothesis. Now, we will verify that $\left\{c l_{M}(D): D \in f^{C}(\mathcal{C})\right\}<<f \mathcal{C}$. (Note that $f$ is a d-map by the assumption). For any $D \in f^{C}(\mathcal{C})$, we have 
$f^{-1}\left[c l_{M}(D)\right] \in \mathcal{C}$, hence, $c l_{M}(D) \supseteq f\left[f^{-1}\left[c l_{M}(D)\right] \in f \mathcal{C}\right.$. Since $B \in \mathcal{C}$ and $B \subseteq f^{-1}[f[B]] \subseteq f^{-1}\left[c l_{M}(f[B])\right]$, we get $f[B] \in f^{C}(\mathcal{C})$.

$\left(\right.$ bcla $\left._{5}\right): D \in f^{C}(\mathcal{C}), D \subseteq c l_{M}(F)$ and $F \in \mathcal{B}^{Y}$ imply $f^{-1}\left[c_{M}(D)\right] \in \mathcal{C}$. But $c l_{M}(D) \subseteq \operatorname{cl}_{M}\left(c_{M}(F)\right) \subseteq \operatorname{cl}_{M}(F)$ imply $f^{-1}\left[c_{M}(D)\right] \subseteq f^{-1}\left[c_{M}(F)\right]$, and $f^{-1}\left[c l_{M}(F)\right] \in \mathcal{C}$ results showing that $F \in f^{C}(\mathcal{C})$. Taking all these facts into account, we conclude that $f^{C}(\mathcal{C})$ defines a $f[B]$-clan in $M$, and thus $f^{C}(\mathcal{C}) \in Y^{C}$ is valid.

(1): Let $A^{C} \subseteq X^{C}, \mathcal{C} \in t_{X^{C}}\left(A^{C}\right)$ and suppose $f^{C}(\mathcal{C}) \notin t_{Y^{C}}\left(f^{C}\left[A^{C}\right]\right)$. Then, $\triangle f^{C}\left[A^{C}\right] \nsubseteq f^{C}(\mathcal{C})$, hence, $D \notin f^{C}(\mathcal{C})$ for some $D \in \triangle f^{C}\left[A^{C}\right]$, which means $f^{-1}\left[c l_{M}(D)\right] \notin \mathcal{C}$. Since $\triangle A^{C} \subseteq \mathcal{C}$, we get $f^{-1}\left[c l_{M}(D)\right] \notin \mathcal{D}$ for some $\mathcal{D} \in A^{C}$. Consequently, $D \notin f^{C}(\mathcal{D})$ results, which is a contradiction because $D \in \triangle f^{C}\left[A^{C}\right]$ is valid.

(2): Now, let $x$ be an element of $X$. We will prove the validity of $f^{C}\left(e_{X}(x)\right)=$ $e_{Y}(f(x))$. To this end, let $D \in e_{Y}(f(x))$. Then, $f(x) \in c_{M}(D)$, hence, $x \in$ $f^{-1}\left[c l_{M}(D)\right]$ and, consequently, $f^{-1}\left[c_{M}(D)\right] \in x_{N}=e_{X}(x)$. Thus, $D \in f^{C}\left(e_{X}(x)\right)$ proving the inclusion $e_{Y}(f(x)) \subseteq f^{C}\left(e_{X}(x)\right)$. We have $\left\{c l_{M}(D)\right.$ : $\left.D \in f^{C}\left(e_{X}(x)\right)\right\}<<f e_{X}(x)$ and, since $f$ is a d-map by the hypothesis, we get $f^{C}\left(e_{X}(x)\right) \in M(\{f(x)\} \backslash\{\emptyset\})$. But $e_{Y}(f(x))$ is maximal in $M(\{f(x)\}) \backslash\{\emptyset\}$ ordered by set inclusion, and the desired equality follows.

Theorem 6.3. We obtain a functor $G: N C L A-S D$ to SYBTEXT by setting:

(a) $G\left(X, \mathcal{B}^{X}, N\right):=\left(e_{X}, \mathcal{B}^{X}, X^{C}\right)$ for any near clan space $\left(X, \mathcal{B}^{X}, N\right)$ with $X:=\left(X, c l_{N}\right)$ and $X^{C}:=\left(X^{C}, t_{X^{C}}\right)$;

(b) $G(f):=\left(f, f^{C}\right)$ for any d-map $f:\left(X, \mathcal{B}^{X}, N\right) \longrightarrow\left(Y, \mathcal{B}^{Y}, M\right)$.

Proof. (a): According to $\left(\operatorname{sd}_{1}\right), c l_{N}: \underline{P} X \longrightarrow \underline{P} X$ is a topological closure operator. Here, we only verify the validity of the axiom $\left(\mathrm{h}_{4}\right)$ in Remark 2.2. Since $x \in c l_{N}\left(c l_{N}(A)\right)$ and $A \in \underline{P} X$ imply $\left\{c l_{N}(A)\right\} \in N(\{x\})$, we have $\{A\} \in N(\{x\})$ by applying $\left(\operatorname{sd}_{8}\right)$ and $\left(\mathrm{b}_{2}\right)$. Thus, $x \in c l_{N}(A)$. Hence, we get the topological closure operator $t_{X^{C}}$ on $X^{C}$. Therefore, we obtain topological spaces with a bornology $\mathcal{B}^{X}$ such that $B \in \mathcal{B}^{X}$ implies $c l_{N}(B) \in \mathcal{B}^{X}$ according to $\left(\operatorname{sd}_{1}\right)$, and $e_{X}: X \longrightarrow X^{C}$ is a function satisfying $\left(\mathrm{btx}_{1}\right)$ and $\left(\mathrm{btx}_{2}\right)$. To prove $\left(\mathrm{btx}_{1}\right)$, let $B \in \mathcal{B}^{X}$ and suppose $x \in c l_{N}(B)$. Then, we get $\triangle e_{X}[B] \subseteq x_{N}$, hence, $e_{X}(x) \in t_{X^{C}}\left(e_{X}[B]\right)$, which means $x \in e_{X}^{-1}\left[t_{X^{C}}\left(e_{X}[B]\right)\right]$. Conversely, let $x$ be an element of $e_{X}^{-1}\left[t_{X^{C}}\left(e_{X}[B]\right)\right]$. Then, by the definition we have $\triangle e_{X}[B] \subseteq x_{N}$. Since $B \in \triangle e_{X}[B]$ we get $x \in \operatorname{cl}_{N}(B)$.

To prove $\left(\mathrm{btx}_{2}\right)$, let $\mathcal{C} \in X^{C}$ and suppose $\mathcal{C} \notin t_{X^{C}}\left(e_{X}[X]\right)$. By the definition, we get $\triangle e_{X}[X] \nsubseteq \mathcal{C}$ so that there exists a set $F \in \triangle e_{X}[X]$ with $F \notin \mathcal{C}$. But then, $X \subseteq c l_{N}(F)$ follows. Since $B \in \mathcal{C}$ for some $B \in \mathcal{B}^{X} \backslash\{\emptyset\}$, we get $B \subseteq c l_{N}(F)$, hence, $F \in \mathcal{C}$ because $\mathcal{C}$ is satisfying (bcla ${ }_{5}$ ). But this is a contradiction and, thus, $\mathcal{C} \in t_{X^{C}}\left(e_{X}[X]\right)$ is valid. To show that $\left(e_{X}, \mathcal{B}^{X}, X^{C}\right)$ is symmetric, let $x$ be an element of $X$ such that $\mathcal{C} \in t_{X^{C}}\left(\left\{e_{X}(x)\right\}\right)$. We have to prove $x_{N} \in t_{X^{C}}(\{\mathcal{C}\})$. By the hypothesis, we have $x_{N} \cap \mathcal{B}^{X} \subseteq \mathcal{C}$, and $\mathcal{C} \in N(B)$ for some $B \in \mathcal{B}^{X} \backslash\{\emptyset\}$. We are to show that $\triangle\{C\} \subseteq x_{N}$. To this end, let $F \in \triangle\{\mathcal{C}\}$. Then, $F \in \mathcal{C} \cap \mathcal{B}^{X}$. We already know that $\{x\} \in \mathcal{C}$. Since $N$ satisfies $\left(q_{N}\right)$, we get $\mathcal{C} \in N(\{x\})$. Hence, $\{F\} \in N(\{x\})$ by $\left(\operatorname{sd}_{3}\right)$, and so $x \in c l_{N}(F)$ is valid showing that $F \in x_{N}$. By the hypothesis, $f:\left(X, \mathcal{B}^{X}, N\right) \longrightarrow\left(Y, \mathcal{B}^{Y}, M\right)$ is a d-map so that $f$ is continuous and 
bi-bounded. It remains to prove that the following diagram commutes:

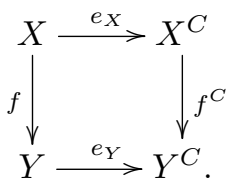

To this end, let $x$ be an element of $X$. We are to show the equality $\left(f^{C} \circ e_{X}\right)(x)=$ $\left(e_{Y} \circ f\right)(x)$.

" $\leq$ ": $D \in\left(f^{C} \circ e_{X}\right)(x)$ implies $D \in f^{C}\left(x_{N}\right)$, which means $f^{-1}\left[c l_{M}(D)\right] \in x_{N}$, hence, $x \in c l_{N}\left(f^{-1}\left[c l_{M}(D)\right]\right)$. Since $f$ is continuous, we have $f(x) \in$ $c l_{M}\left(f\left[f^{-1}\left[c l_{M}(D)\right]\right]\right) \subseteq c l_{M}(D)$, and $D \in f(x)_{M}=e_{Y}(f(x))$ follows.

" $\geq ": D \in e_{Y}(f(x))$ implies $f(x) \in c l_{M}(D)$, hence, $x \in f^{-1}\left[c_{M}(D)\right]$ follows, and $x \in c l_{N}\left(f^{-1}\left[c l_{M}(D)\right]\right.$ is valid. Thus, $f^{-1}\left[c l_{M}(D)\right] \in e_{X}(x)$, which means $D \in f^{C}\left(e_{X}(x)\right)$. Thus, the composition of d-maps is preserved by $G$. Finally, we will show that the image of $G$ is also contained in the full subcategory STBTEXT of BTEXT whose objects are the strict bornotopological extensions.

Corollary 6.4. The image of $G$ is contained in STBTEXT.

Proof. Consider $\mathcal{C} \notin X^{C}$ and let $A^{C}$ be closed in $X^{C}$ with $\mathcal{C} \notin A^{C}$. Then, $\mathcal{C} \notin t_{X^{C}}\left(A^{C}\right)$, hence, $\triangle A^{C} \nsubseteq \mathcal{C}$. We can find some $F \in \triangle A^{C}$ such that $F \notin \mathcal{C}$. Now, for each $\mathcal{D} \in A^{C}$ we have $F \in \mathcal{D}$, which implies $\triangle e_{X}[F] \subseteq \mathcal{D}$ and, therefore, $\mathcal{D} \in t_{X^{C}}\left(e_{X}[F]\right)$. On the other hand, since $F \notin \mathcal{C}$, we have $\triangle e_{X}[F] \nsubseteq \mathcal{C}$, hence, $\mathcal{C} \notin t_{X^{C}}\left(e_{X}[F]\right)$, which proves the statement.

Theorem 6.5. Let $H: S Y B T E X T \longrightarrow N C L A-S D$ and $G: N C L A-S D \longrightarrow$ STBTEXT be the above defined functors. For each object $\left(X, \mathcal{B}^{X}, N\right)$ of NCLA$S D$, let $t_{\left(\mathcal{B}^{X}, N\right)}$ denote the identity map $i d_{X}: H\left(G\left(X, \mathcal{B}^{X}, N\right)\right) \longrightarrow\left(X, \mathcal{B}^{X}, N\right)$. Then, $t: H \circ G \longrightarrow 1_{N C L A-S D}$ is a natural equivalence from $H \circ G$ to the identity functor $1_{N C L A-S D}$, i.e., id $X: H\left(G\left(X, \mathcal{B}^{X}, N\right)\right) \longrightarrow\left(X, \mathcal{B}^{X}, N\right)$ is d-map in both directions for each object $\left(X, \mathcal{B}^{X}, N\right)$, and the following diagram commutes for each d-map $f:\left(X, \mathcal{B}^{X}, N\right) \longrightarrow\left(Y, \mathcal{B}^{Y}, M\right)$ :

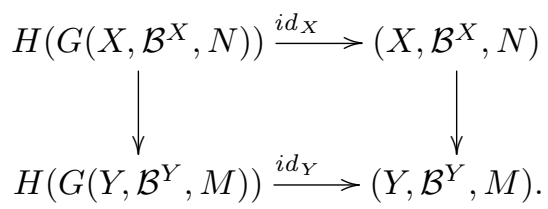

Proof. The commutativity of the diagram is obvious because $H(G(f))=f$. It remains to prove that $i d_{X}: H\left(G\left(X, \mathcal{B}^{X}, N\right)\right) \longrightarrow\left(X, \mathcal{B}^{X}, N\right)$ is a d-map in both directions. Since $H\left(G\left(X, \mathcal{B}^{X}, N\right)\right)=\left(X, \mathcal{B}^{X} N^{e_{X}}\right)$ by the definitions of $G$ and $H$, it suffices to show that for each $B \in \mathcal{B}^{X} \backslash\{\emptyset\}$ we have $N^{e_{X}}(B) \subseteq N(B) \subseteq N^{e_{X}}(B)$. To this end, assume $\rho \in N^{e_{X}}(B)$. Then, there exists $\mathcal{C} \in t_{X^{C}}\left(e_{X}[B]\right)$ such that $\rho \in \bigcap\left\{t_{X^{C}}\left(e_{X}[F]\right): F \in \rho \cap \mathcal{B}^{X}\right\}$. Hence, $\triangle e_{X}[B] \subseteq \mathcal{C}$. We get $B \in \mathcal{C}$ and $\mathcal{C} \in$ $N\left(B_{1}\right)$ for some $B_{1} \in \mathcal{B}^{X} \backslash\{\emptyset\}$. Since $N$ satisfies $\left(q_{N}\right)$, we obtain $\left\{B_{1}\right\} \cup \mathcal{C} \in N(B)$ with $\mathcal{C}<<\left\{B_{1}\right\} \cup \mathcal{C}$, hence, $\mathcal{C} \in N(B)$. But $A \in \rho \cap \mathcal{B}^{X}$ implies $\mathcal{C} \in t_{X^{C}}\left(e_{X}[A]\right)$, hence, $\triangle e_{X}[A] \subseteq \mathcal{C}$. Since $A \in \triangle e_{X}[A]$, we get $A \in \mathcal{C}$, and $\rho \cap \mathcal{B}^{C} \subseteq \mathcal{C}$ results. Consequently, $\rho \cap \mathcal{B}^{X} \in N(B)$ is valid implying $\rho \in N(B)$ according to $\left(\operatorname{sd}_{2}\right)$. 
Conversely, let $\rho \in N(B)$. Since $\left(X, \mathcal{B}^{X}, N\right)$ is a clan space, we can choose a Bclan $\mathcal{C}$ in $N$ such that $\rho \cap \mathcal{B}^{X} \subseteq \mathcal{C}$. In order to show $\rho \in N^{e_{X}}(B)$ we need to verify

(1) $\mathcal{C} \in t_{X C}\left(e_{X}[B]\right)$

(2) $\mathcal{C} \in \bigcap\left\{t_{X^{C}}\left(e_{X}[F]\right): F \in \rho \cap \mathcal{B}^{X}\right\}$.

(1): Let $D$ be an element of $\triangle e_{X}[B]$. Then, $B \subseteq c l_{N}(D)$, which implies $D \in \mathcal{C}$ according to $\left(\right.$ bcla $\left._{4}\right)$ and (bcla 5$)$.

(2): Let $F$ be an element of $\rho \cap \mathcal{B}^{X}$. We are to show that $\triangle e_{X}[F] \subseteq \mathcal{C}$. $A \in \triangle e_{X}[F]$ implies $F \subseteq c l_{N}(A)$. By the hypothesis, $F \in \mathcal{C}$ implies $A \in \mathcal{C}$ according to (bclas), which concludes the proof.

Corollary 6.6. For a btop- $T_{1}$ extension $E:=\left(e, \mathcal{B}^{X}, Y\right)$ where $e$ is a topological embedding and $Y$ is a $T_{1}$-space, $\left(X, \mathcal{B}^{X}, N^{e}\right)$ is separated by satisfying

(sep) $x, z \in X$ and $\{\{z\}\} \in N^{e}(\{x\})$ imply $x=z$.

Proof. Let $\{\{z\}\} \in N^{e}(\{x\})$ Then, there exists $y \in t_{Y}(\{e(x)\}) \cap t_{Y}(\{e(z)\})$. By the hypothesis, $e(x)=y=e(z)$, hence, $x=z$ because $e$ is injective.

Corollary 6.7. For a separated sd-space $\left(X, \mathcal{B}^{X}, N\right)$, the function $e_{X}: X \longrightarrow$ $X^{C}$ is injective.

Proof. For $x, z \in X$, let $e_{X}(x)=e_{X}(z)$ Then, $z \in c l_{N}(\{x\})$, and thus $\{\{x\}\} \in$ $N(\{z\})$. By the hypothesis, $x=z$ is valid.

Remark 6.8. To make Theorem 6.5 more transparent, we claim that a near sdspace $\left(X, \mathcal{B}^{X}, N\right)$ is a clan space if and only if it can be regarded as a subspace of a topological space $Y$ such that the B-near collections in $N$ are characterized by the fact that the meet of their closures of bounded members in $Y$ meets the closure of $B$ in $Y$. In the case when $\mathcal{B}^{X}$ is saturated, that means $X \in \mathcal{B}^{X}$, the near sd-spaces essentially coincide with the nearness spaces up to isomorphism. Hence, the above theorem generalizes one of Bentley presented by him in [3] where bunch-determined nearness spaces are playing an important role. With respect to Section 3, it is also possible to define corresponding b-contiguities or b-proximities, respectively and to consider their related topological extensions. But these attempts seem to be superflous because we would get special cases of Theorem 6.5. The above mentioned facts also immediately concern the still more specific spaces such as the contiguity spaces or the proximity spaces and their related extensions studied by several authors in the past (see References). So, we have found a unique topological concept, by which all these previously considered cases can be easily described.

\section{REFERENCES}

[1] B. Banaschewski, Extensions of topological spaces, Canad. Math. Bull. 7 (1964), 1-23.

[2] R. Bartsch, Compactness Properties for Some Hyperspaces and Function Spaces, Shaker Verlag, Aachen, 2002.

[3] H. L. Bentley, Nearness spaces and extension of topological spaces, in: N. Stavrakas and K. Allen (eds.), Studies in Topology, Academic Press, NY, 1975, 47-66. 
[4] J. Deák, Extending a family of screens in a contiguity space, in: Á Császár (ed.), Topology with Applications, Proceedings of the 7th Colloquium, Szekszárd, Hungary, 1993, Bolyai Soc. Math. Stud. 4 (1995), 121-133.

[5] G. Dimov and E. Ivanova, On some categories arising in the theory of locally compact extensions, Math. Pannon. 19 (2008), 219-239.

[6] D. Doîtchînov, Compactly determined extensions of topological spaces, Serdica Bulgaricae Math. Pub. 11 (1985), 269-286.

[7] H. Herrlich, A concept of nearness, General Topology Appl. 4 (1974), 191-212.

[8] H. Hogbe-Nlend, Bornologies and Functional Analysis, North-Holland, Amsterdam, 1977.

[9] M. Hušek, Categorical connections between generalized proximity spaces and compactifications, Contributions to Extension Theory of Topological Structures (Proc. Symp. Berlin 1967), Deutscher Verlag der Wissenschaften, Berlin, 1969, 127-132.

[10] V. M. Ivanova and A. Ivanov, Contiguity spaces and bicompact extensions, Dokl. Akad. Nauk SSSR 127 (1959), 20-22.

[11] D. C. Kent and W. K. Min, Neighbourhood spaces, Int. J. Math. Math. Sci. 32 (2002), 387399.

[12] D. Leseberg, Supernearness, a common concept of supertopologies and nearness, Topology Appl. 12 (2002), 145-156.

[13] D. Leseberg, Bounded topology: A convenient foundation for topology, FU Berlin, 2009, http://www.digibib.tu-bs.de/?docid=00029438.

[14] D. Leseberg, Improved nearness research, Adv. Pure. Math. 4 (2014), 610-626.

[15] D. Leseberg, Improved nearness research II, Rostock. Math. Kolloq. 66 (2011), 87-102.

[16] D. Leseberg, Improved nearness research III, Int. J. Math. Sci. Appl. 1 (2011), 1-14.

[17] D. Leseberg, Improved nearness research IV, Quaest. Math. 37 (2014), 249-263.

[18] D. Leseberg, Bounded proximities and related nearness, Quaest. Math. 36 (2013), 381-387.

[19] M. W. Lodato, On topologically induced generalized proximity relations II, Pacific J. Math. 17 (1966), 131-135.

[20] S. Naimpally, Proximity Approach to Problems in Topology and Analysis, Oldenbourg Wissenschaftsverlag, München, 2009.

[21] H. Poppe, On locally defined topological notions, Questions Answers Gen. Topology 13 (1995), 39-53.

[22] Y. M. Smirnov, On the completeness of proximity spaces (in Russian), Dokl. Akad. Nauk. SSSR 88 (1953), 761-794.

Dieter Leseberg, Department of Mathematics and Informatics, Free University of Berlin, Arnimallee 14, 14195 Berlin, Germany

e-mail: leseberg@zedat.fu-berlin.de 
